\title{
Estimation of Bit Error Rates for Narrow-Band Digital Communication in the Presence of Atmospheric Radio Noise Bursts
}

\author{
A. R. K. SASTRY
}

\begin{abstract}
The lack of representativeness in the existing analysis concerning the problem of estimation of bit error rates for narrowband digital communications in the presence of atmospheric burst noise, arising due to the omission of consideration of the structure and time parameters of the noise bursts, is emphasized. A method of analysis in which these parameters can suitably be introduced is indicated. Areas in which further noise data is to be acquired are identified.
\end{abstract}

\section{INTRODUCTION}

The effect of atmospheric radio noise on the performance of narrow-band digital communication systems was considered by several workers [1]. However, the parameters of the noise used in the analyses are hardly representative of the interfering character of the noise since the structure and time characteristics of the noise were ignored. Further, the variation of error rates with operational frequency and bandwidth under constant signal conditions cannot be evaluated by merely using the relevant amplitude probability distributions of the noise since the structure of the noise changes with frequency and bandwidth.

This paper is prepared to bring out the role of information on structure and time characteristics of the noise in the estimation of bit error probabilities in narrow-band binary digital communcation.

\section{Characteristics of Atmospheric Noise Bursts}

A. Structure of the Noise Bursts

The detected output of a narrow-band receiver that picks up radiated noise due to a complete lightning discharge is

Paner aonroved by the Radio Communication Committee of the IEEE Communication Technology Group for publication without oral presentation. Manuscript received December 23,1970 ; revised May $24,1971$.

The author is with the Department of Electrical Communication Engineering, Indian Institute of Science, Bangalore 12, India. 

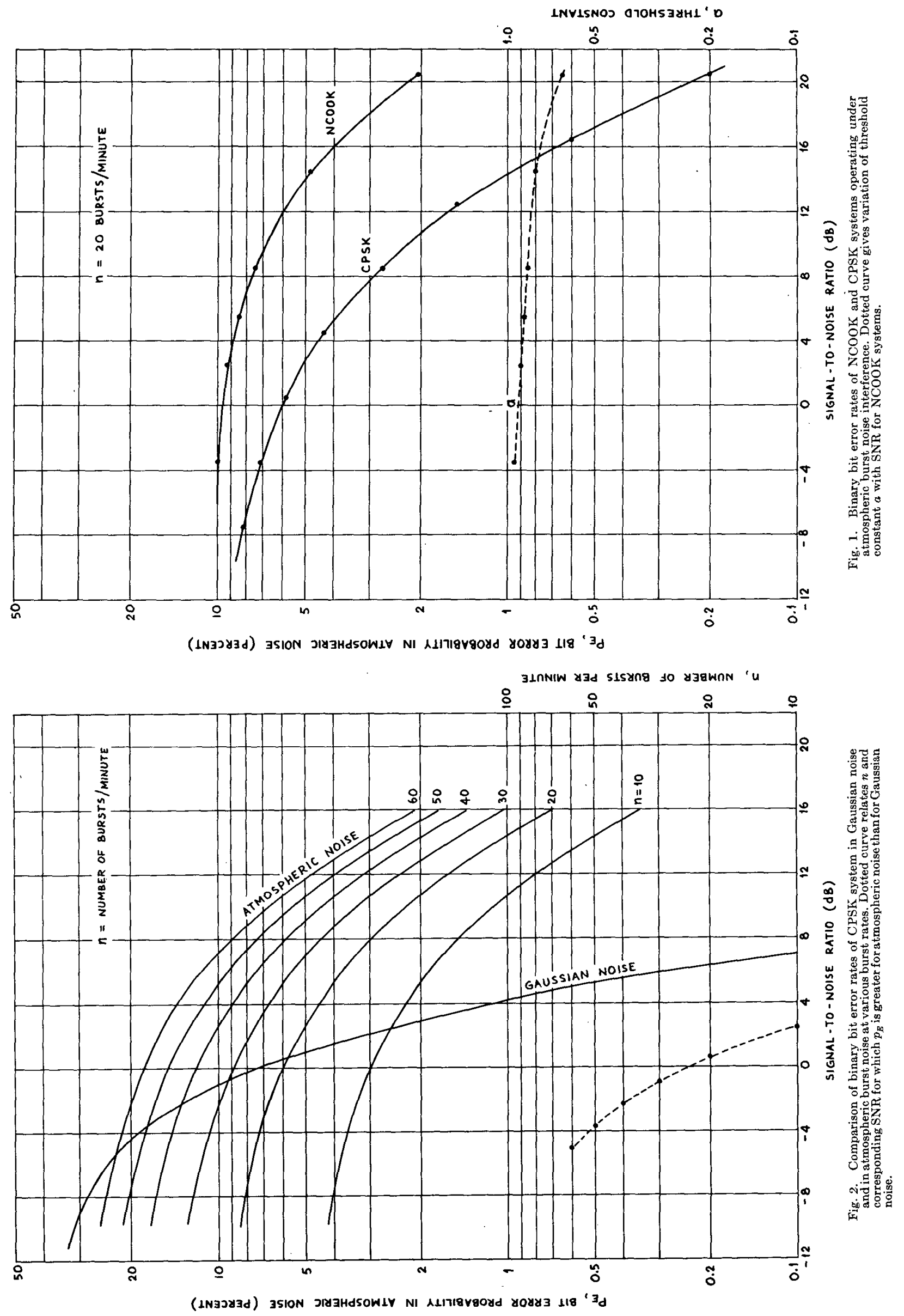
called a noise burst. The noise burst is composed of. distinct pulses of varying amplitude with duration of a pulse being determined by the bandwidth of the receiver. The number of pulses in a specified range of level from the peak pulse amplitude increases with frequency [2], [3] in the frequency range in which atmospheric noise is considered the principal source of interference, i.e., up to $30 \mathrm{MHz}$.

\section{B. Short-Term Time Parameters of Noise Bursts [4], [5].}

The duration of a noise burst changes randomly. Short-term studies on noise bursts arising from local thunderstorms show that the duration $t_{d}$ is $\log$-normally distributed with a median $t_{\mathrm{d} m}$ of $500 \mathrm{~ms}$ and standard deviation of $\mathbf{0 . 6 3}$ natural $\log$ units. Short-term studies on noise burst rate (number of bursts per minute) $n$ revealed that the burst repetition period $t$, is also $\log$-normally distributed with median $t_{r n t}$ of $60 / n \mathrm{~s}$ and standard deviation of 0.44 natural $\log$ units. Long-term data on $n$ is available for tropical regions.

\section{Estimation of Bit ERror Rates}

Consider the situation in which atmospheric noise is the principal source of interference and assume that errors can occur in the reception only during the presence of noise bursts. The noise bursts occur in a sequence with random intervals between the bursts. At the transmission speeds that are commonly in practice, several signal symbols occur within the duration of a burst. All the signal symbols (each signal symbol may be represented by several cycles of modulated sinusoid) will not be corrupted to the same extent due to the random variation in the amplitudes of the pulses and random occurrences of pulses themselves within the burst. Due to the large number of signal symbols that occur within the duration of the burst (150-1500 symbols for a 1000-baud transmission), a statistical investigation can be attempted to yield a probability of binary bit error $p_{o}$ of receiving a binary symbol in error within the burst, A prerequisite for such an analysis is the amplitude distribution $p(N)$ of the noise pulses within a burst at the operating frequency and bandwidth.

$p_{e}$ for systems using different types of modulation of a sinusoidal carrier can be evaluated easily with the help of phasor diagrams, on the lines indicated by Montgomery [6] and Engel [7]. However, $p_{e}$ does not fully represent the probability of error during the presence of a burst, since the burst is composed of distinct pulses and regions characterized by their absence. From the knowledge of the number of pulses in a burst, the time occupied by the pulses in the burst $t_{0}$ can be calculated. If a reasonable assumption that the pulses are distnbuted uniformly in the burst is made, then the mean value of $t_{\alpha} / t_{d}$ gives probability $p_{p}$ that each signal symbol meets with a noise pulse.

The overall bit error probability $P_{t}$ in the presence of a sequence of distinct noise bursts is given by

$$
p_{E}=p_{e} \cdot p_{p} \cdot \alpha
$$

where $\alpha$ is the fraction of time occupied by the bursts in the total time of a long sequence, equal to $\bar{t}_{d} / \bar{t}_{r}$.

Data on long term variation of the burst occurrence rate $n$ are available for tropical locations. $p_{p}$ is to be evaluated from knowledge of the number of pulses in the noise bursts and is a function of operating frequency. $p$, for HF bands tends to be nearly equal to unity since pulses occur fairly continuously throughout the bursts. However, in the lower frequency bands the pulses are distinct and widely separated, occupying insignificant portions of burst duration, and leading to extremely small values of $p$. . Thus, under the same signal level conditions, as the operating frequency is lowered, the increase in $p_{e}$ due to higher noise levels is counteracted by the reduction in $p$, ..

Error rate calculations are carried out for noncoherent on-off keying $(\mathrm{NCOOK})$ and coherent phase-shift keying (CPSK) systems on the lines indicated above, using noise data available for India [8].

\section{Noise Data}

Season: March-May - time-block, 20-24 h

Frequency: $1 \mathrm{MHz}$ - bandwidth, $1 \mathrm{kHz} / 6 \mathrm{~dB}$

Seasonal median noise level: $30 \mathrm{~dB}$ above $1 \mu \mathrm{V} / \mathrm{m}$.

Short-term median noise level: $27 \mathrm{~dB}$ approximately.

Short-term noise level distribution: $\log$ normal.

Standard deviation: $8 \mathrm{~dB}$.

Short-term median peak amplitude: $32 \mathrm{~dB}$.

Short-term median rms amplitude: $29.5 \mathrm{~dB}$.

$p_{p} \approx \mathbf{1}, \quad \boldsymbol{n}=\mathbf{2 0}$.

\section{CONCLuSions}

The results are plotted in Fig. 1, in which SNR is the ratio of squares of rms signal level and median rms noise level. In the NCOOK case, the optimum value for threshold constant $a$ for which $P_{E}$ is minimum, is a function of SNR, and is also plotted in the same figure. $a$ is greater than $\mathbf{1 / 2}$ even for SNR as high as $20 \mathrm{~dB}$. Thus, the arbitrary choice of $a$ as $\mathbf{1 / 2}$ for large SNR as was done in Montgomery's analysis [6] in the calculation of $P_{E}$ versus SNR does not hold for atmospheric noise.

It is interesting to compare the error probabilities due to atmospheric noise with that of Gaussian noise. Fig. 2 gives the curves $p_{E}$ versus SNR for CPSK signaling in atmospheric burst noise environment for different burst rate $\boldsymbol{n}$ and in Gaussian noise. Using these curves, another curve relating to $\boldsymbol{n}$ and corresponding SNR for which $p_{E}$ for atmospheric noise $\left(p_{E A}\right)$ is greater than $p_{E}$ for Gaussian noise $\left(p_{E G}\right)$ is plotted in the same figure. As $n$ increases, SNR for which $p_{E A}$ is greater than $p_{E G}$ decreases. An important, conclusion that can be drawn from this curve is that the interference from atmospheric noise is more severe than from Gaussian noise of the same rms level, for predetection SNR. greater than about $2 \mathrm{~dB}$.

The analysis described in this paper breaks down for the LF band since statistical analysis within the burst to arrive at $p_{e}$ does not hold, due to the very small number of pulses observable in the bursts. In addition, since the dynamic range of the amplitudes of pulses is rather large in this frequency region, amplitude limiting occurs in the preliminary wide-band stages of the receiver. This limiting effect is to be taken into account to link the predetection SNR with receiver input SNR.

\section{ACKNOWLEDGMENT}

The author is grateful to Prof. S. V. C. Aiya and Prof. B. S. Ramakrishna for their encouragement and keen interest in the work.

\section{REFERENCES}

[1] P. A. Bello and R. Esposito, "A new method for calculating probabilities of errors due to impulsive noise" IEE E Trans. Commun. Technol..,vol. COM-17, June 1969 , pp. 368-379.

T. Pierce, "Radio emissions from close lightning," in Planetary Electrodynamics. S. C. Coroniti and J. Hughes, Eds. New York:

31 D. J. Malan. "Radiation from lightning discharges and its relation to the discharge process," in Rrcent Advances in Atmospheric Electricity, G. Smith, Ed. New York: Pergamon Press, 1959, Ap. 557-563.

[4] S. V. C. Aiya, "Some characteristics of tropical thunderstorms," Nature. vol. 208, h'ov. 1965, pp. 641-644.

[5] S. V. C. Aiya and $\mathrm{K}$. $\mathrm{N}$. Lakshminarayan, "Atmospheric radio noise bursts in the LF band at Bangalore," J. Res. Nat. Bur. Stand., Sect. D, vol. 69, Oct. 1965. $\mathrm{pp}$. 1351-1358.

[6] $\boldsymbol{G}$. Montgomery, "A comparison of amplitude and angle modulation for narrowband communication of binary-coded messages in fluctuation noise," Proc. $I R E$. vol. 42 , Feb. 1954 , pp. $447-454$.

[7] J. S. Engel, "Digital transmission in the presence of impulsive noise." Bell Syst. Tech. J., vol. 44, Oct. 1965, pp. 1699-1743.

S. V. C. Aiya. "MF and HF band atmospheric noise data for India." $J$. Inst. Telecommun. Eng. New Delhi, vol. 15, Feb. 1969, pp. 144-157. 